University of Wollongong

Research Online

Faculty of Engineering - Papers (Archive)

Faculty of Engineering and Information

Sciences

November 1993

\title{
Fermi-energy edge singularity and excitonic enhancement associated with the second subband in asymmetric modulation-doped quantum wells
}

\author{
T. A. Fisher \\ University of Wollongong \\ Philip E. Simmonds \\ University of Wollongong, simmonds@uow.edu.au \\ M. S. Skolnick \\ University of Sheffield, UK
}

A. D. Martin

University of Wollongong

R. S. Smith

G.E.C. Marconi Caswell Technology Ltd, UK

Follow this and additional works at: https://ro.uow.edu.au/engpapers

Part of the Engineering Commons

https://ro.uow.edu.au/engpapers/268

\section{Recommended Citation}

Fisher, T. A.; Simmonds, Philip E.; Skolnick, M. S.; Martin, A. D.; and Smith, R. S.: Fermi-energy edge singularity and excitonic enhancement associated with the second subband in asymmetric modulationdoped quantum wells 1993.

https://ro.uow.edu.au/engpapers/268

Research Online is the open access institutional repository for the University of Wollongong. For further information contact the UOW Library: research-pubs@uow.edu.au 


\title{
Fermi-energy edge singularity and excitonic enhancement associated with the second subband in asymmetric modulation-doped quantum wells
}

\author{
T. A. Fisher \\ Department of Physics, University of Wollongong, Wollongong, New South Wales 2500, Australia \\ and Department of Physics, University of Sheffield, Sheffield S3 7RH, United Kingdom \\ P. E. Simmonds \\ Department of Physics, University of Wollongong, Wollongong, New South Wales 2500, Australia \\ M. S. Skolnick \\ Department of Physics, University of Sheffield, Sheffield S3 7RH, United Kingdom \\ A. D. Martin \\ Department of Physics, University of Wollongong, Wollongong, New South Wales 2500, Australia \\ R. S. Smith \\ G.E.C.-Marconi Caswell Technology Ltd., Caswell, Towcester NN12 8EQ, United Kingdom \\ (Received 22 February 1993; revised manuscript received 7 June 1993)
}

\begin{abstract}
A study of the Fermi-energy edge singularity (FEES) in the second $(n=2)$ subband of asymmetric modulation-doped (AlGa)As-(InGa)As-GaAs quantum wells (AMDQW's) is reported. In one of the AMDQW's a Schottky gate is employed to vary the electron density in the $n=2$ subband $\left(n_{s, 2}\right)$ from 0 to $1 \times 10^{11} \mathrm{~cm}^{-2}$. Temperature-dependent photoluminescence excitation (PLE) measurements clearly show that the $n=2$ PLE feature has FEES character for $n_{s, 2} \gtrsim 0.4 \times 10^{11} \mathrm{~cm}^{-2}$. In contrast to PLE, photoluminescence $(\mathbf{P L})$ intensity is not a true measure of oscillator strength, since PL intensity can be affected by competing recombination pathways. Temperature-dependent PL measurements have been performed on two types of AMDQW. One type has $n_{s, 2} \sim 0$, with the Fermi energy close to the $n=2$ subband energy. The other type has $n_{s, 2}=1 \times 10^{11} \mathrm{~cm}^{-2}$ and a FEES associated with $n=2$ observed in PLE. We demonstrate that the very similar broadening and reduction in peak height of the $n=2$ PL peak with temperature for the two types of samples can be accounted for in terms of spreading of the electron or exciton populations near the $n=2$ subband edge. Therefore, we conclude that temperature-dependent PL does not provide unequivocal evidence for a many-body enhancement of the $n=2$ PL transition, in contrast to that reported by Chen et al. [Phys. Rev. Lett. 64, 2434 (1990)].
\end{abstract}

\section{INTRODUCTION}

Modulation-doped heterojunctions ${ }^{1}$ and quantum wells (MDQW's) are particularly suitable for the study of many-electron effects in the optical properties of semiconductor heterostructures. The spatial separation of the high-density two-dimensional electron gas (2DEG) from the dopant atoms has allowed a variety of manybody effects to be studied with minimal perturbation from the donor impurities. Several properties related to the many-body character of the 2DEG, can be investigated optically. These include band-gap renormalization, ${ }^{2-4}$ Fermi-energy edge singularity (FEES), ${ }^{5-10,4,11-16}$ "shake-up" processes, ${ }^{15,16}$ magnetoexciton effects ${ }^{17}$ and collective interactions in the fractional quantized Hall regime. ${ }^{18,19}$

The FEES is observed in photoluminescence (PL) spectra as an enhancement of oscillator strength for transitions close to the Fermi level, $E_{F}$, in MDQW's where disorder or hole localization allows wave-vectornonconserving transitions to occur. ${ }^{5}$ In optical absorp- tion, or photoluminescence excitation (PLE), the FEES appears as an excitonic enhancement at the threshold of transitions into unoccupied states above $E_{F}$. The occurrence of the FEES is due to correlation between the photoexcited hole and the high-density Fermi sea of electrons. ${ }^{11-14}$ A characteristic feature of the FEES enhancement is a strong decrease in oscillator strength and substantial broadening with temperature up to $\sim 50$ $\mathrm{K}^{5-10,12}$ The strong temperature dependence arises from broadening of the Fermi surface and consequent weakening of the electron-hole correlation with increasing temperature. Typically the FEES is observed in absorption as an excitonic enhancement of a factor of $\sim 2$ at $4 \mathrm{~K}$ for carrier densities of the order of $1-5 \times 10^{11} \mathrm{~cm}^{-2}$, weakening at higher densities. ${ }^{6-9,12,13}$

Many of the previous experimental and theoretical studies of the FEES have dealt with structures where only the lower $n=1$ electron subband of the $\mathrm{QW}$ is significantly occupied. ${ }^{6-8}$ In most of these cases the Fermi energy, $\varepsilon_{F}$ is much less than the energy separation $\left(E_{2}-E_{1}\right)$ of the two lowest subbands. More recent work 
on the FEES has involved structures where two subbands are occupied. Of particular interest is the dependence of the FEES associated with the second, $n=2$, electron subband on both $n=2$ carrier density and the position of $E_{F}$ relative to the $n=2$ subband edge. Experimental work, both with ${ }^{21-24}$ and without ${ }^{10}$ magnetic field, and theoretical studies ${ }^{25-27}$ have been reported.

In the present paper, a photoluminescence (PL) and photoluminescence excitation (PLE) study, at zero magnetic field, of excitonic processes associated with $n=2$ subbands in doped QW's is reported. The samples used in our study are a series of $\mathrm{Al}_{y} \mathrm{Ga}_{1-y} \mathrm{As}-\mathrm{In}_{x} \mathrm{Ga}_{1-x} \mathrm{As}-$ $\mathrm{GaAs}$ wide asymmetric modulation-doped strained layer quantum wells (AMDQW's) with varying well width $(w \sim 150 \AA-250 \AA)$ and doping (electron density $n_{s}$ between $\left.0.8 \times 10^{12}-1.6 \times 10^{12} \mathrm{~cm}^{-2}\right)$. The electron Fermi energy $\left(\varepsilon_{F}\right)$ ranges between greater than and slightly less than the separation $\left(E_{2}-E_{1}\right)$ between the $n=1,2$ subbands. In our previous experiments ${ }^{10}$ FEES character in $n=2$ was only seen in PLE for samples where the $n=2$ population was significant, in spite of the large $n=1$ population present in all the AMDQW's.

In the present work a Schottky gate is applied to one of the QW structures of well width $250 \AA$. This enables a systematic study to be made of the effect of varying the carrier density in the $n=2$ subband from zero up to $\sim 1 \times 10^{11} \mathrm{~cm}^{-2}$, while avoiding sample to sample variability. Utilizing the characteristic temperature dependence of the FEES, we determine a minimum value $n_{s, 2} \gtrsim 0.4 \times 10^{11} \mathrm{~cm}^{-2}$ at which the fully developed FEES sets in. The $n=2$ carrier densities are estimated from observed variations in the effect of band filling on the PL linewidth and (when possible) Landau-level depopulation effects on magneto-PL spectra.

The present and previous work ${ }^{10}$ has shown that temperature-dependent studies of excitonic peaks in absorption (or PLE) can provide unequivocal identification of such peaks as originating from "atomic" exciton or FEES processes. In AMDQW's, PL associated with the $n=2$ electron subband $\left(E_{21}\right)$ is also observed to be enhanced by a factor of $\sim 2$ by electron-hole Coulomb interactions after oscillator strength modifications due to the electron-hole wave-function overlaps have been accounted for. The nature of this PL enhancement is also dependent on the $n=2$ population. Chen et al. ${ }^{21}$ have presented results on structures, where only a single peak, $E_{21}$, is observed at $E_{F}$, in which the decrease in peak height and broadening of the $E_{21}$ PL peak with increasing temperature was interpreted as evidence for a FEES. In the present work careful temperature-dependent measurements of the $E_{21}$ and $E_{11} \mathrm{PL}$ are performed on two types of AMDQW's where $E_{F}$ is close to $E_{2}$. From the first type, with $E_{F}$ slightly greater than $E_{2}$, a FEES is observed in the $E_{21}$ PLE (labeled $E_{21}^{*}$ ); in the other, with $E_{F}$ slightly less than $E_{2}$, the $E_{21}^{*} \quad$ PLE is of atomic exciton nature. In contrast to the PLE results the $E_{21}$ PL from both types of samples shows very similar temperature-dependent broadening and decrease in peak amplitude which are interpreted in terms of changes in the electron and exciton distributions. We thus conclude that observation of temperature-dependent $E_{21}$ PL cannot necessarily be regarded as evidence for a many-body related mechanism such as the FEES. Preliminary temperature-dependent PL and PLE measurements on the Schottky gated structure have been reported by us previously. ${ }^{28}$

The paper is organized in the following way. In the next section details of the structures and of the experiments are given. Section III provides a description of the PL spectra from the samples studied. Then in Section IV the temperature dependence of the PLE spectra as a function of applied bias on a Schottky gated structure is described. Section $V$ discusses the significance of temperature-dependent broadening of $E_{21}$ PL from samples with differing $n=2$ populations. The main conclusions are summarized in Sec. VI.

\section{EXPERIMENTAL DETAILS}

The $\mathrm{Al}_{y} \mathrm{Ga}_{1-y} \mathrm{As}-\mathrm{In}_{x} \mathrm{Ga}_{1-x} \mathrm{As}-\mathrm{GaA}$ A AMDQW's were grown by molecular-beam epitaxy ${ }^{29}$ and consisted of the following layers grown on a semi-insulating GaAs substrate: $1-\mu \mathrm{m}$ undoped $\mathrm{GaAs}, \mathrm{In}_{x} \mathrm{Ga}_{1-x}$ As quantum well $(x \sim 0.1$, thickness $w)$, undoped $\mathrm{Al}_{y} \mathrm{Ga}_{1-y} \mathrm{As}(y \sim 0.23)$ spacer (thickness $d_{1}$ ), $\mathrm{Al}_{y} \mathrm{Ga}_{1-y}$ As doped region (thickness $d_{2}$, doping level $n_{2}$ ) and GaAs capping layer $\left(d_{3}, n_{3}\right)$. The In contents $(x)$ in the QW layers were estimated from the growth conditions ${ }^{29}$ and are consistent with the measured PL energies, after allowing for the effects of band bending and band-gap renormalization. ${ }^{30,3,10}$ Some of the details of the individual samples are given in Tables I and II; also included are the total electron sheet carrier density $\left(n_{s}\right)$, together with $E_{2}-E_{1}$,

TABLE I. Structural parameters for the (AlGa)As-(InGa)As-GaAs asymmetric modulation-doped quantum well structures (AMDQW's). The AMDQW's consist of the following layers grown on a semi-insulating GaAs substrate: $1-\mu \mathrm{m}$ undoped $\mathrm{GaAs}, \mathrm{In}_{x} \mathrm{Ga}_{1-x}$ As quantum well (thickness, $w$ ), undoped $\mathrm{Al}_{y} \mathrm{Ga}_{1-y} \mathrm{As}\left(y=0.23\right.$ ), spacer (thickness $\left.d_{1}\right), \mathrm{Al}_{y} \mathrm{Ga}_{1-y}$ As doped region [thickness, $d_{2}$; doping,

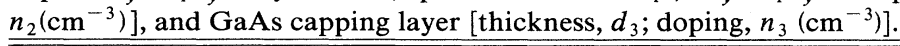

\begin{tabular}{lccccccc}
\hline \hline Sample & $\begin{array}{c}w \\
(\AA)\end{array}$ & $\begin{array}{c}d_{1} \\
(\AA)\end{array}$ & $\begin{array}{c}d_{2} \\
(\AA)\end{array}$ & $\begin{array}{c}n_{2} \\
\left(\mathrm{~cm}^{-3}\right)\end{array}$ & $\begin{array}{c}d_{3} \\
(\AA)\end{array}$ & $\begin{array}{c}n_{3} \\
\left(\mathrm{~cm}^{-3}\right)\end{array}$ & $x$ \\
\hline$A$ & 250 & 100 & 250 & $1.5 \times 10^{18}$ & 200 & $1 \times 10^{14}$ & $0.10(5)$ \\
$B$ & 200 & 40 & 400 & $1 \times 10^{18}$ & 100 & $2 \times 10^{18}$ & $0.07(5)$ \\
$C$ & 150 & 40 & 400 & $5 \times 10^{18}$ & 100 & $5 \times 10^{18}$ & $0.07(5)$ \\
$D$ & 150 & 40 & 400 & $1.5 \times 10^{18}$ & 400 & $2 \times 10^{18}$ & $0.07(5)$ \\
\hline \hline
\end{tabular}


TABLE II. Electronic data for the AMDQW's of well width $(w)$ and total electron concentration in the well of $n_{s}\left(\mathrm{~cm}^{-2}\right)$. The data displayed are the experimentally obtained and calculated values of the Fermi energy, $\varepsilon_{F}$, the energy position of the Fermi level, $E_{F}$ above the second electron subband (energy $\left.E_{2}\right), E_{F}-E_{2}$, and the energy separation of the electron subbands $E_{2}-E_{1}$. Also shown is the $E_{11} \mathrm{PL}$ transition energy between the first electron and first hole subband. $n_{s}$ values were obtained from Shubnikov-de Haas and PL data.

\begin{tabular}{lcccccr}
\hline \hline Sample & $\begin{array}{c}w \\
(\AA)\end{array}$ & $\begin{array}{c}n_{s} \\
\left(\mathrm{~cm}^{-2}\right)\end{array}$ & $\begin{array}{c}\varepsilon_{F} \\
(\mathrm{meV})\end{array}$ & $\begin{array}{c}E_{F}-E_{2} \\
(\mathrm{meV})\end{array}$ & $\begin{array}{c}E_{2}-E_{1} \\
(\mathrm{meV})\end{array}$ & $\begin{array}{c}E_{11} \\
(\mathrm{eV})\end{array}$ \\
\hline$A$ & 250 & $1 \times 10^{12}$ & 30.5 & 3.5 & 27.0 & 1.402 \\
$B$ & 200 & $1 \times 10^{12}$ & 34.0 & $\sim 0$ & 34.5 & 1.407 \\
$C$ & 150 & $1.61 \times 10^{12}$ & 56.0 & $\sim 0$ & 56.0 & 1.403 \\
$D$ & 150 & $1.43 \times 10^{12}$ & 49.0 & -5 & 54.0 & 1.407 \\
\hline \hline
\end{tabular}

the electron Fermi energy $\varepsilon_{F}$ and the energy position of the electron Fermi level, $E_{F}$, above the $n=2$ subband, $E_{F}-E_{2}$. These values were determined from magneto photoluminescence (magneto-PL) and Shubnikov-de Haas (SdH) measurements. Care was taken that the carrier density employed in interpreting the data corresponds to the particular portion of the wafer and to similar illumination conditions used for the optical experiments. Additional details of layer growth and structure can be found in Refs. 10 and 29. Semitransparent gold Schottky barriers of $100-\AA$ thickness were evaporated on to the top surface of a portion of sample $A$, and Ohmic contact was made to the substrate. By varying the bias the $n=2$ electron density $n_{s, 2}$ (and $n_{s}$ ) could be controlled.

PL and PLE measurements were carried out at temperatures between 2 and $50 \mathrm{~K}$ in a variable temperature flow cryostat. The photoluminescence was excited by either a tunable Ti:sapphire laser, Styryl-9 dye laser, or fixed wavelength $\mathrm{He}-\mathrm{Ne}$ laser. The emitted radiation was dispersed with a $0.75-\mathrm{m}$ double grating, or $1-\mathrm{m}$ single grating spectrometer and detected by a cooled $77-\mathrm{K}$ Ge photodiode.

\section{PHOTOLUMINESCENCE OF THE AS-GROWN SAMPLES}

The PL spectra at low excitation between 2 and $4 \mathrm{~K}$ for the four principal samples studied in detail ( $A, B, C$, $D)$ are shown in Figs. 1(a)-1(d). The results of PL measurements on samples $A$ and $B$ have been discussed in detail elsewhere. ${ }^{10}$ The spectra generally consist of two bands, labeled $E_{11}$ and $E_{21}$; these arise from recombination of holes thermalized in the $n=1$ heavy-hole valence subband (HH1) with, respectively, electrons in $n=1$ and either in, or very close in energy to, $n=2$ conduction subbands. ${ }^{10,21,29,31}$ Sample $D$ [Fig. 1(d)] shows only $E_{11}$ PL. The $E_{11}$ recombination peaks near the $n=1$ subband edge $(k=0)$ and exhibits a strong decrease in intensity as it extends towards $E_{F}$. These transitions are weakly allowed since the in plane wave-vector selection rule $(\Delta k=0)$ is broken by weak disorder (or hole localization). $5,10,37,32$ This contrasts with the case of significant disorder where strong luminescence is observed at $E_{F} \cdot{ }^{33}$ For samples $A, B$, and $C$ the relative peak height of $E_{21}$

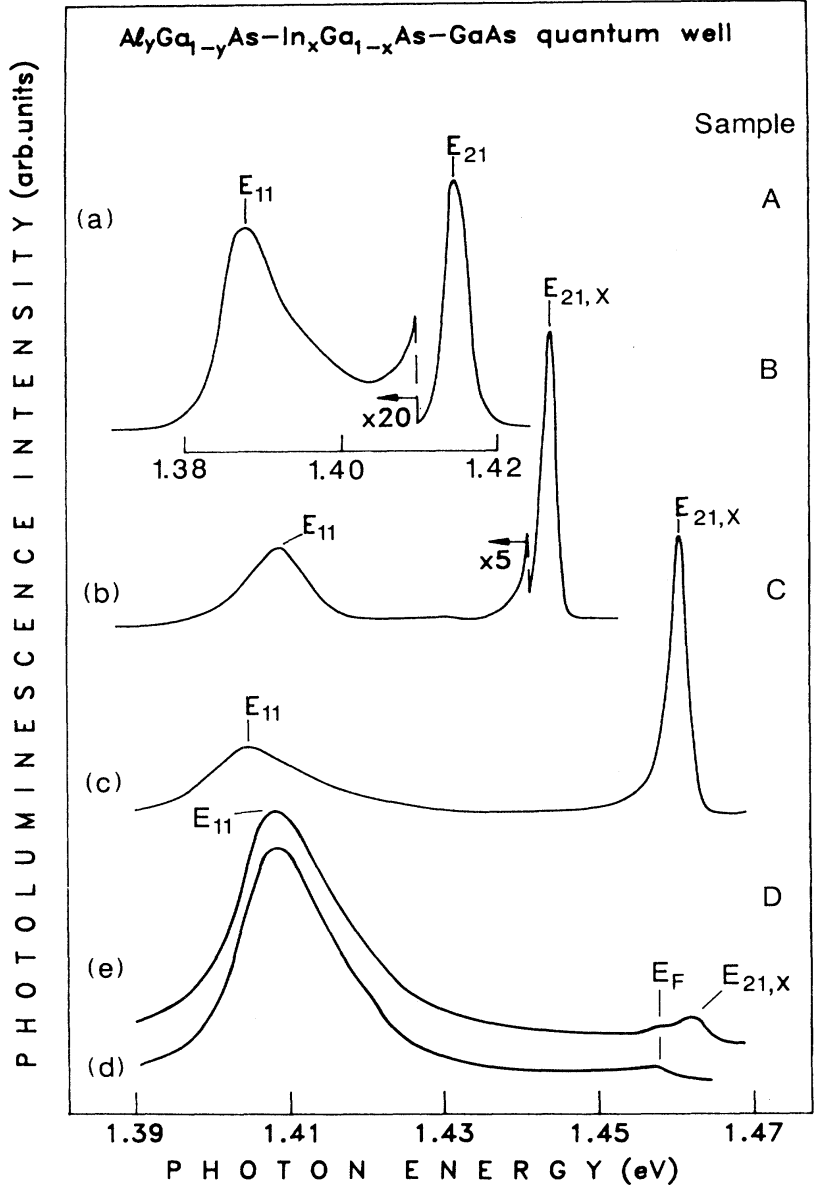

FIG. 1. PL spectra measured from the AMDQW's. For (a) sample $A$, (b) sample $B$, and (c) sample $C$ at $T=4 \mathrm{~K}$ both peaks $E_{21}$ and $E_{11}$ are observed, arising from recombination of electrons in the $n=1$ and $n=2$ electron subband, respectively, with photoexcited thermalized holes in the first heavy hole subband. In (b) sample $B$ and (c) sample $C$ the $E_{21}$ recombination is of atomic exciton nature and is labeled $E_{21, X}$. (d) Only $E_{11}$ recombination is observed from sample $D$ at $T=4 \mathrm{~K}$ with a weak enhancement at $E_{k}$ attributed to hybridized mixing with $n=2$ states. (e) At $T=12 \mathrm{~K}$ the hybridized peak at $E_{F}$ and weak $E_{21, X}$ recombination are observed. 
to $E_{11}$ recombination ranges from a factor $\sim 3$ for sample $B$ (well width $w=150 \AA$ ) to $\sim 22$ for sample $A$ $(w=250 \AA)$. This is due to two factors. First, in asymmetric QW's at high $n_{s}$, the electrostatic band-bending potential leads to localization of $n=1$ electrons and HH1 holes at opposite interfaces of the QW. ${ }^{3,10}$ Selfconsistent (Hartree) solutions of Poisson's and Schrödinger's equations for sample $C$ predict a ratio of $E_{21}$ to $E_{11}$ band-to-band oscillator strengths of 3, increasing up to 11 in sample A. ${ }^{10}$ Second, $E_{21}$ transitions experience excitonic enhancement of $\sim 2$ to 3 . This enhancement may be of atomic exciton or FEES character as discussed below. The combination of these two effects has been shown to account well for the observed $E_{21}$ to $E_{11}$ intensities in Figs. 1(a)-1(c). The experimentally determined sample parameters, discussed below, are in good agreement with values obtained from selfconsistent calculations.

For the as-grown sample $A$, the electron subband separation $E_{2}-E_{1}=27.0 \mathrm{meV}$ corresponds approximately to the difference in energies between the $E_{21}$ and $E_{11}$ transition peaks [Fig. 1(a)] and is determined from extrapolation to zero field of $n=1,2$ Landau level (LL) transitions observed in magneto-PL. From Shubnikov-de Haas (SdH) measurements $\varepsilon_{F, 1}=30.5 \mathrm{meV}$ so that the Fermi level $E_{F}$ resides $3.5 \mathrm{meV}$ above the bottom of the $n=2$ subband. The $n=2$ Fermi energy, $\varepsilon_{F, 2}=3.5 \mathrm{meV}$ is consistent with $n_{s, 2}=(1 \pm 0.1) \times 10^{11} \mathrm{~cm}^{-2}$ obtained from the depopulation of $E_{21}$ Landau levels in a magnetic field and with the observation of a low-field series in $\mathrm{SdH}$ measurements.

Figure 1(b), $c$ shows the 2-K PL for samples $B$ and $C$, of well widths 200 and $150 \AA$, respectively, for which $E_{F}$ resides close to energy $E_{2}$. No LL splitting or narrowing of $E_{21}$ transitions is observed in this case, allowing an upper limit of $\sim 1.0 \mathrm{meV}$ to be placed on $\varepsilon_{F, 2}$, corresponding to $n_{s, 2} \lesssim 0.3 \times 10^{11} \mathrm{~cm}^{-2}$. Even though the $n=2$ population is small, $E_{21}$ is observed close to its full oscillator strength relative to $E_{11} ;{ }^{10}$ varying the laser excitation over more than an order of magnitude causes only weak changes in relative intensities. In magnetic field, the $n=2$ PL transitions have definite exciton character; consequently the labeling $E_{21, X}$ will be employed. In zero magnetic field the excitonic enhancement associated with $n=2$ can be attributed to either of the two mechanisms described below.

First, the recombination could be of an atomic exciton nature, which involves a small real population of $n=2$. A simulation of the PL emission line shape, indicates that the $E_{21}$ PL transitions would attain their full peak height, at low temperature, for $n_{s, 2} \gtrsim 0.3 \times 10^{11} \mathrm{~cm}^{-2}$. (Further details of the line-shape simulations are given in the following section.) This value of $n_{s, 2}$ is consistent with the upper limit given above and also with the observed atomic exciton nature of $n=2 E_{21, X}$ absorption peaks measured in PLE. ${ }^{10}$ Second, in the absence of real $n=2$ population where $E_{F}$ is less than but within the excitonic binding energy of $E_{2}$ the enhancement can be interpreted as a many-body excitonic interaction. This interaction leads to hybridization, in the presence of photocreated holes, between $n=1$ electron states near the sharp Fermi edge at $E_{F}$ and $n=2$ states with $k \simeq 0 .^{22,23,25-27}$ The $k=0$ exciton character and enhanced electron-hole overlap for $E_{21}$, relative to $E_{11}$, would contribute to the relatively large oscillator strength of the hybridized excitonic peak in the same manner as expected for excitonic recombination associated with a small "real" $n=2$ population.

For sample $D$, where only $E_{11}$ recombination is observed and $E_{F}$ is $5 \mathrm{meV}$ below $E_{2}$ a small peak is observed in the PL spectrum at $E_{F}$ [Fig. $\left.1(\mathrm{~d}), T=4 \mathrm{~K}\right]$. At elevated temperature, $T \gtrsim 10 \mathrm{~K}$ [shown at $T=12 \mathrm{~K}$, Fig. $1(\mathrm{e})]$, or under increased laser excitation, a weak peak associated with $n=2\left(E_{21, X}\right)$ is observed in addition to the enhancement at $E_{F}$. The small enhancement at $E_{F}$ at both 4 and $12 \mathrm{~K}$ can be attributed to the hybridized exciton mechanism, as described first by Chen and coworkers $^{22,23}$ for GaAs-(AlGa)As and (AlGa)As(InGa)As-GaAs MDQWs and discussed by Hawrylak. ${ }^{26}$ It is only in this case, where two spectroscopic features are observed, that PL associated with the two mechanisms of the hybridized exciton and the $n=2$ atomic excitonic enhancement can be distinguished.

\section{THE EFFECT OF CARRIER DENSITY ON THE OPTICAL TRANSITIONS}

The principal sample used in this aspect of the study was the as-grown and Schottky gated sample $A$ with $w=250 \AA$ (see Table I). PL at low excitation (dashed line) and PLE (full line) spectra at $T \sim 4 \mathrm{~K}$ and higher temperatures are illustrated in Fig. 2 for sample $A$. The spectra are shown for the as-grown structure [Fig. 2(a)] and for reverse biases $V_{b}$, applied to the gated structure, of $V_{b}=0.3$ and $0.7 \mathrm{~V}$ (Figs. 2(b) and 2(c)]. The $E_{21} \mathrm{PL}$ peak for both the as-grown and gated samples $\left(V_{b}=0\right)$ is broadened due to band filling by $n=2$ electrons. ${ }^{33}$ With increasing reverse bias $V_{b}$ the PL linewidth narrows from $4.5 \mathrm{meV}$ in the as-grown sample to $2.5 \mathrm{meV}$ at $V_{b} \simeq 0.6$ $\mathrm{V}$ corresponding to a decrease in $n_{s, 2}$ from $1 \times 10^{11} \mathrm{~cm}^{-2}$ to $0\left(\varepsilon_{F}=E_{2}-E_{1}\right)$. Over the same bias range the lower subband is depleted from $n_{s, 1} \sim 9 \times 10^{11} \mathrm{~cm}^{-2}$ to $n_{s, 1} \sim 5.5 \times 10^{11} \mathrm{~cm}^{-2}$, as determined from the change in $\varepsilon_{F}$ which follows the decrease in separation of the $E_{21}$ and $E_{11}$ peaks with $V_{b}$. At $V_{b}=0.7 \mathrm{~V}$ [Fig. 2(c)] the presence of a Fermi-energy edge in the $E_{11}$ PL recombination $\sim 3.5 \mathrm{meV}$ below $E_{2}$, and the very weak intensity of $E_{21}$ transitions (reduced by a factor of 10 ), indicates that $E_{F}<E_{2}$ and $n_{s, 2} \sim 0$. The persistence of $E_{21} \mathrm{PL}$ at this bias is attributed to sample inhomogeneity (the $E_{21}$ relative intensity does not change appreciably over the range of excitation from $4-300 \mathrm{~mW} / \mathrm{cm}^{2}$ ).

The PLE spectrum of the as-grown sample is shown at $4 \mathrm{~K}$ in Fig. 2(a). A well-defined excitonic peak (linewidth $4.5 \mathrm{meV}$ ), labeled $E_{21}^{*}$, is observed at $E_{F}$, corresponding to transitions into empty states in the $n=2$ subband. $E_{22}$, involving excitation of an electron from $\mathrm{HH} 2$ valence to $n=2$ electron subband states is also observed, together with other transitions between higher electron and hole subbands. ${ }^{10}$ Figure $3(\mathrm{a})$ illustrates that at $4 \mathrm{~K}$, $E_{21}^{*}$ shows little variation relative to its continuum over 
the range of bias studied on the gated sample $\left(V_{b}=0-0.7\right.$ V) corresponding to an $n=2$ population varying between $0.5 \times 10^{11} \mathrm{~cm}^{-2}$ to $\sim 0$. (The determination of $n_{s, 2}$ is given below). The as-grown $\left(n_{s, 2} \simeq 1 \times 10^{11} \mathrm{~cm}^{-2}\right)$ [Fig. 2(a)] and gated sample at $V_{b}=0 \mathrm{~V}$ [see Fig. 3(a)] also show very similar $E_{21}^{*}$ absorption. The excitonic peak height enhancement over the continuum (PHE) of $E_{21}^{*}$ is a factor of 2 over this range, comparable to excitons of similar linewidth in undoped QW's. ${ }^{20}$ With increasing $V_{b}$ (decreasing $n_{s, 2}$ ) both $E_{21}^{*}$ and $E_{22}$ become slightly narrower [Fig. 3(a)], where the linewidth of $E_{21}^{*}$ varies from $4.5 \mathrm{meV}$ for the as-grown sample to $2.2 \mathrm{meV}$ at $V_{b}=0.7$ $\mathrm{V}\left(n_{s, 2} \sim 0\right)$; asymmetric broadening with increasing $n_{s}$ is expected behavior for FEES resonances. ${ }^{6,12}$ Also the strength of $E_{22}$ increases relative to $E_{21}^{*}$ with $V_{b}$ as expected from self-consistent calculations; the initially strong band bending in the QW weakens, thus increasing

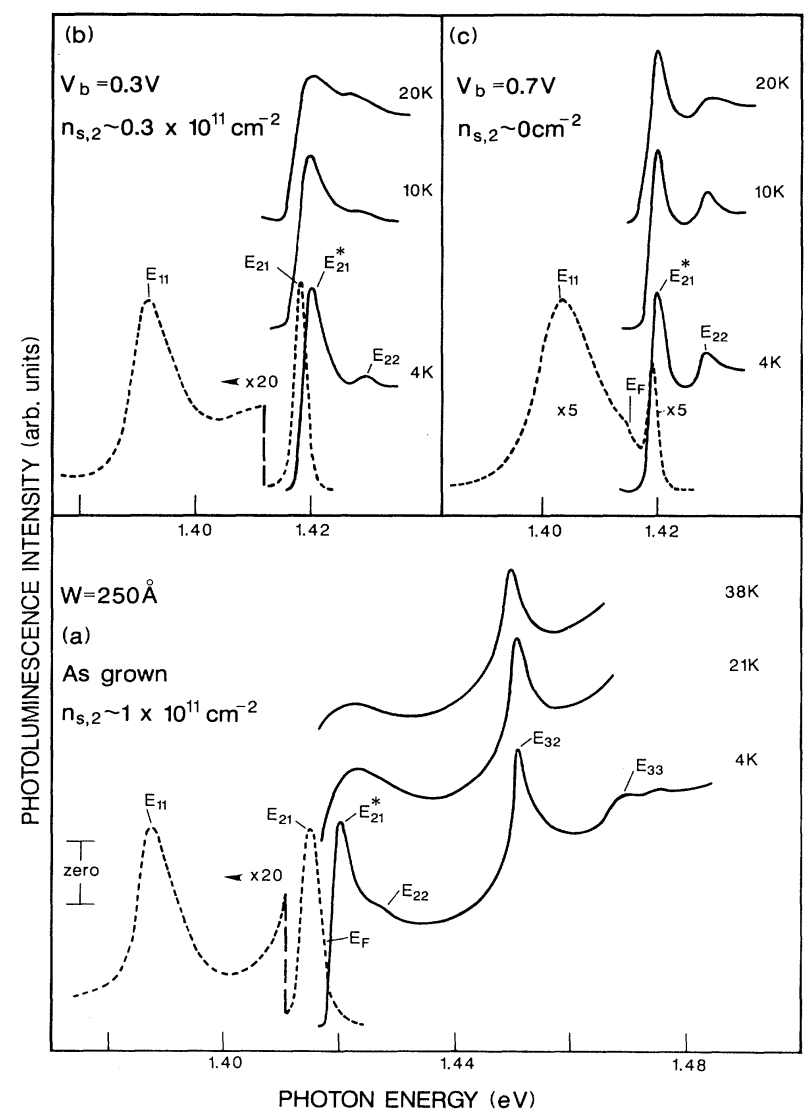

FIG. 2. PL (dashed line) at $T=4 \mathrm{~K}$ and PLE (full line) for a range of temperatures 4 to $40 \mathrm{~K}$. For (a) the as-grown sample $A$ and (b) the Schottky gated sample $A$ at reverse bias $V_{b}=0.3 \mathrm{~V}$ the excitonic peak associated with $n=2, E_{21}^{*}$ is strongly broadened and weakened with temperature, clearly identifying $E_{21}^{*}$ as a Fermi-energy edge singularity. (c) At $V_{b}=0.7 \mathrm{~V}$ the $E_{21}^{*}$ PLE transition is of atomic exciton nature since the strength of $E_{21}^{*}$ is not affected by temperature. This is consistent with $E_{21}$ in PL being of reduced intensity in comparison with (a) and (b). the overlap of wave functions of electrons and holes of the same subband index, while also bringing about the reduction in $E_{2}-E_{1}$.

The temperature dependence of the PLE spectra is shown in Figs. 2(a)-2(c). Most strikingly in Figs. 2(a) and 2(b), where $n=2$ has significant population (low $V_{b}$ ), the $E_{21}^{*}$ peak weakens and shows strong asymmetric broadening with increasing temperature up to and beyond about $20 \mathrm{~K}$. By contrast, this effect is much weaker for the spectra at $V_{b}=0.7 \mathrm{~V}$ in Fig. 2(c) where $n=2$ is largely depleted. Figure $3(\mathrm{~b})$ shows the PLE spectra measured at $T=20 \mathrm{~K}$ (full line) and $T=40 \mathrm{~K}$ (dashed line) for the same biases shown in Fig. 3(a) at $T=4 \mathrm{~K}$. Comparison of Figs. 3(a) and 3(b) illustrates very clearly the significantly different behavior of the $E_{21}^{*}$ PLE peak at elevated temperatures when compared to the 4-K PLE spectra. For biases up to $V_{b}=0.3 \mathrm{~V}$ weakening and broadening of $E_{21}^{*}$ is clearly seen at $T \sim 20 \mathrm{~K}$ [full line, Fig. 3(b)]. By $T \sim 40 \mathrm{~K}$ [dashed line, Fig. 3(b)] the original sharp $E_{21}^{*}$ feature is present only as a very weak enhancement. For $V_{b} \geq 0.6 \mathrm{~V}$ [shown at $V_{b}=0.7$ and $0.95 \mathrm{~V}$, Fig. 3(b)], where $n=2$ is depleted, the temperature dependence is very weak.

The above features are shown quantitatively in Fig. 4 where the temperature dependence of the $E_{21}^{*}$ peak height above the continuum (relative to its value at $4 \mathrm{~K}$ ) is plotted against temperature at various $V_{b}$. In the intermediate bias region between 0.3 and $0.6 \mathrm{~V}, E_{21}^{*}$ exhibits a decrease in peak height with temperature [shown in Fig. 3(b) and Fig. 4]. However, the broadening of the peak is much weaker than for $V_{b} \leq 0.3 \mathrm{~V}$. Thus at higher temperatures marked changes in the $E_{21}^{*}$ PLE spectra are observed as a function of $V_{b}$, in contrast with the weak variation with $V_{b}$ observed at $4 \mathrm{~K}$.

In order to interpret these results in detail, it is necessary to determine the $n=2$ population $n_{s, 2}$, as a function of $V_{b}$. The change in line shape of $E_{21}$ PL recombination at $4 \mathrm{~K}$ was used to estimate $n_{s, 2}$ vs $V_{b}$ up to $V_{b}=0.5 \mathrm{~V}$, based on the assumption of simple band-to-band recombination, in a similar manner to that described in Ref. 34 . The line-shape fitting procedure involved the convolution of a Gaussian line (to account for broadening) with Fermi and Boltzmann distribution functions for electrons and photocreated holes, respectively, ${ }^{35,36}$ together with a decreasing transition probability towards the Fermi energy (fall-off factor). The fitting parameters were determined predominantly from the $n=2$ PL spectrum of the asgrown sample, shown in Fig. 5(a) (full line). As discussed in Sec. III, for sample A depopulation of $n=2$ Landau levels in magneto-optical spectra and magneto transport measurements give $n_{s, 2}=(1 \pm 0.1) \times 10^{11} \mathrm{~cm}^{-2} .33,34,10$ The Gaussian width was determined from the magneticfield spectrum at $B=2.5 \mathrm{~T}$ (only $N_{e}=0 \mathrm{LL}$ is occupied), shown in Fig. 5(d) with substantial narrowing of linewidth to $1.7 \mathrm{meV}$ from $4.5 \mathrm{meV}$ at zero field. The hole temperature and fall-off factor were obtained from a combination of fitting both the zero-field spectra and the spectra in magnetic field for $B<2.5 \mathrm{~T}$ for the as-grown sample. The LL relative intensities at these $B$ values, where more than one $n=2 \mathrm{LL}$ is occupied, are well ac- 
counted for by the parameters. For the gated sample at $V_{b}=0 \mathrm{~V}, n_{s, 2}=(0.5 \pm 0.1) \times 10^{11} \mathrm{~cm}^{-2}$ was obtained from the zero magnetic field line shape. The close fit to the experimental spectra obtained from the line-shape parameters is shown in Figs. 5(a) and (b) (dashed line) for $n_{s, 2} \simeq 1 \times 10^{11} \mathrm{~cm}^{-2}$ (as grown) and $n_{s, 2} \simeq 0.5 \times 10^{11} \mathrm{~cm}^{-2}$ $\left(V_{b}=0 \mathrm{~V}\right)$, respectively.

The variation of $n_{s, 2}$ vs $V_{b}$ deduced from the line-shape fitting is shown in the inset to Fig. 3(a). Below $n_{s, 2} \sim 0.3 \times 10^{11} \mathrm{~cm}^{-2}$ the band-filling contribution to the broadening becomes too small to allow estimation of $n_{s, 2}$. For $V_{b}=0.5 \mathrm{~V}, n_{s, 2}$ is estimated to be between 0 and $0.3 \times 10^{11} \mathrm{~cm}^{-2}$ (some broadening is still observed). $V_{b}=0.6 \mathrm{~V}$ is the highest bias at which the peak height of $E_{21}$ is at full strength relative to that of $E_{11}$. Also, the $E_{21}$ spectral width is at its narrowest. Hence, at this bias $\varepsilon_{F}$ is estimated to be approximately equal to $E_{2}-E_{1}$. For biases beyond $V_{b}=0.6 \mathrm{~V}$ the PL intensity of $E_{21}$ decreases sharply indicating that $\varepsilon_{F}<E_{2}-E_{1}$ and $n=2$ is depopulated.

The Moss-Burstein shift $\left[\varepsilon_{F, 2}\left(1+m_{e} / m_{h}\right)\right]$ (Refs. 3, 9, and 10) between $n=2$ PL and PLE was used to provide a separate estimate of $n_{s, 2}$. For the as-grown sample and at $V_{b}=0 \mathrm{~V}$ Stokes shifts of $\Delta_{s}=5$ and $3 \mathrm{meV}$, respectively, are found; these Stokes shifts correspond to $n_{s, 2}=0.95 \times 10^{11}$ and $0.55 \times 10^{11} \mathrm{~cm}^{-2}$, in good agreement with the values determined from the line-shape analysis.

In summary, the PLE spectra in Fig. 3(b) show that pronounced broadening and weakening of the $E_{21}^{*}$ excitonic enhancement with increasing temperature, which is the characteristic signature of the FEES, is clearly observed for $V_{b}<0.3 \mathrm{~V}$; this corresponds to carrier densities $n_{s, 2}(4 \mathrm{~K}) \gtrsim 0.3 \times 10^{11} \mathrm{~cm}^{-2}$ A small increase of $n_{s, 2}$ with temperature due to thermal excitation of electrons from $n=1$ raises this value to $n_{s, 2}(20 \mathrm{~K}) \gtrsim 0.4 \times 10^{11}$ $\mathrm{cm}^{-2}$ at $20 \mathrm{~K}$, the temperature at which the quenching of the FEES actually occurs. In the region $0.3 \mathrm{~V}<V_{b}<0.6$
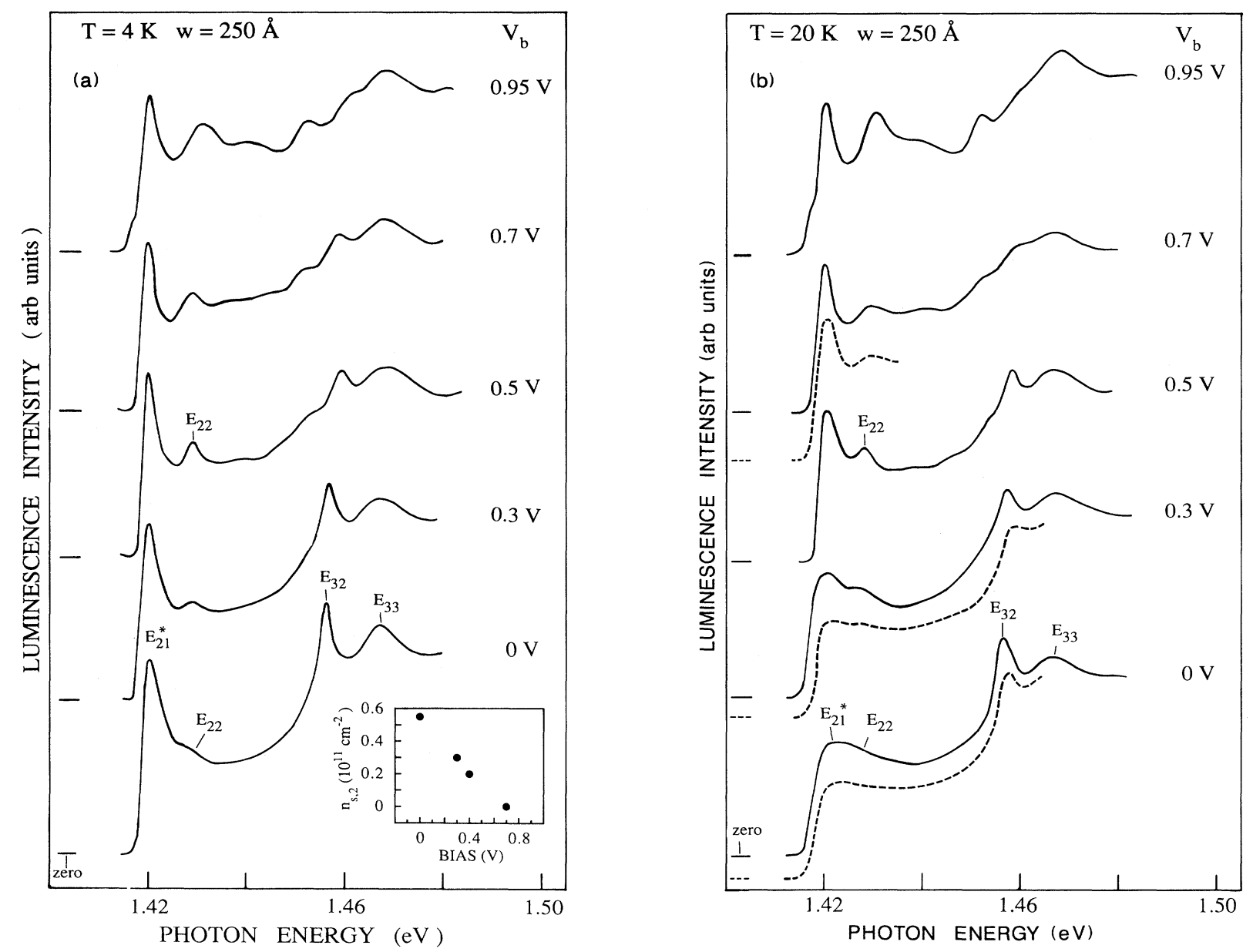

FIG. 3. (a) PLE at $T=4 \mathrm{~K}$ for a range of reverse biases, $V_{b}$, applied to the gated sample $A$, where carrier density is reduced from $n_{s, 2} \sim 0.5 \times 10^{11} \mathrm{~cm}^{-2}$ at $V_{b}=0 \mathrm{~V}$ to $n_{s, 2} \sim 0$ at $V_{b}>0.7 \mathrm{~V}$. The variation of $n_{s, 2}$ with $V_{b}$ is plotted in the inset. (b) PLE at the same biases, $V_{b}$, measured at $T=20 \mathrm{~K}$ (full line) and $T=40 \mathrm{~K}$ (dashed line). In contrast to $T=4 \mathrm{~K}, E_{21}^{*}$ varies from a broad peak of reduced amplitude, at $V_{b}=0 \mathrm{~V}$ to a sharp excitonic peak at $V_{b}>0.7 \mathrm{~V}$. 


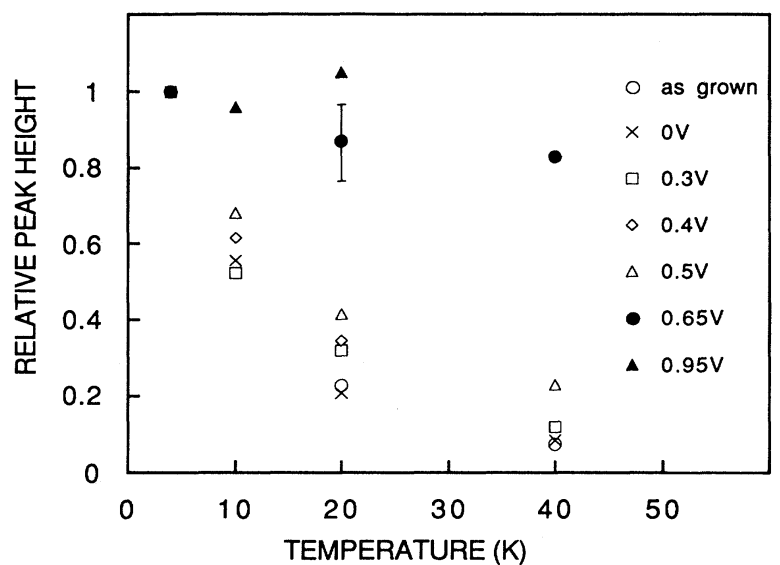

FIG. 4. The $E_{21}^{*}$ peak height measured from the continuum, relative to its $4-\mathrm{K}$ value, is plotted against temperature. Open circles denote the as-grown sample $A$ and the other symbols denote the Schottky gated sample $A$ at various reverse biases, $V_{b}$. At biases $V_{b}<0.3 \mathrm{~V}$ for $T>20 \mathrm{~K}$ the peak height of $E_{21}^{*}$ relative to its $4-\mathrm{K}$ value is strongly reduced.

$\mathrm{V}\left[n_{s, 2}(4 \mathrm{~K}) \lesssim 0.3 \times 10^{11} \mathrm{~cm}^{-2}\right.$ but greater than zero] a transition takes place from fully developed FEES towards atomic exciton behavior. When $V_{b}=0.6 \quad \mathrm{~V}$ $\left(E_{F} \simeq E_{2}-E_{1}\right)$, the weak temperature dependence of $E_{21}^{*}$ in PLE is similar to that of atomic exciton transitions associated with the higher subbands and to that seen in undoped QW's. This behavior is consistent with that found for samples $\mathrm{B}$ and $\mathrm{C}$ where $\varepsilon_{F} \simeq E_{2}-E_{1}{ }^{10}$ For $V_{b}>0.6$ $\mathrm{V}\left(n_{s, 2} \simeq 0\right), n=2$ is depopulated and the atomic exciton behavior of the $E_{21}^{*}$ transition is observed, as expected.

The temperature-dependent behavior of $n=2$ is similar to experiments on QW's having low $n_{s}$ values and only the $n=1$ subband occupied. Characteristic FEES behavior was reported by Saker et al. ${ }^{7}$ down to $n_{s, 1} \simeq 0.3 \times 10^{11} \mathrm{~cm}^{-2}$ in an (InGa)As-InP QW (110 ̊) and by Lee, Miura, and Ando for $n_{s, 1} \simeq 1.4 \times 10^{11} \mathrm{~cm}^{-2}$ but not for $0.5 \times 10^{11} \mathrm{~cm}^{-2}$ in (AlGa)As-GaAs QW's (250 and $84 \AA$ ). Thus, the present experiments provide clear evidence that the presence of a high density of electrons in $n=1$ has no significant effect on the FEES in the $n=2$ subband. FEES behavior is observed only in the presence of appreciable $n_{s, 2}$ values, at $n_{s, 2} \gtrsim 0.4 \times 10^{11} \mathrm{~cm}^{-2}$, consistent with the results published earlier for the $n=1$ subband case.

\section{TEMPERATURE DEPENDENCE OF $n=2$ PL TRANSITIONS}

The temperature variation of $n=2$ related PL transitions (denoted generally as $E_{21}$ ) for samples $A, B$, and $C$ is described in this section. In Figs. 6(a)-6(d) PL spectra for sample $A\left(n_{s, 2} \simeq 1 \times 10^{11} \mathrm{~cm}^{-2}\right)$ are shown for a range of temperatures, $T=4-60 \mathrm{~K}$, the temperature range over which FEES behavior of $E_{21}$ is clearly observed in PLE. Both $E_{21}$ and $E_{11}$ PL peaks are displayed in Fig. 6, showing the $\sim 20$ times stronger peak amplitude of $E_{21}$ rela-

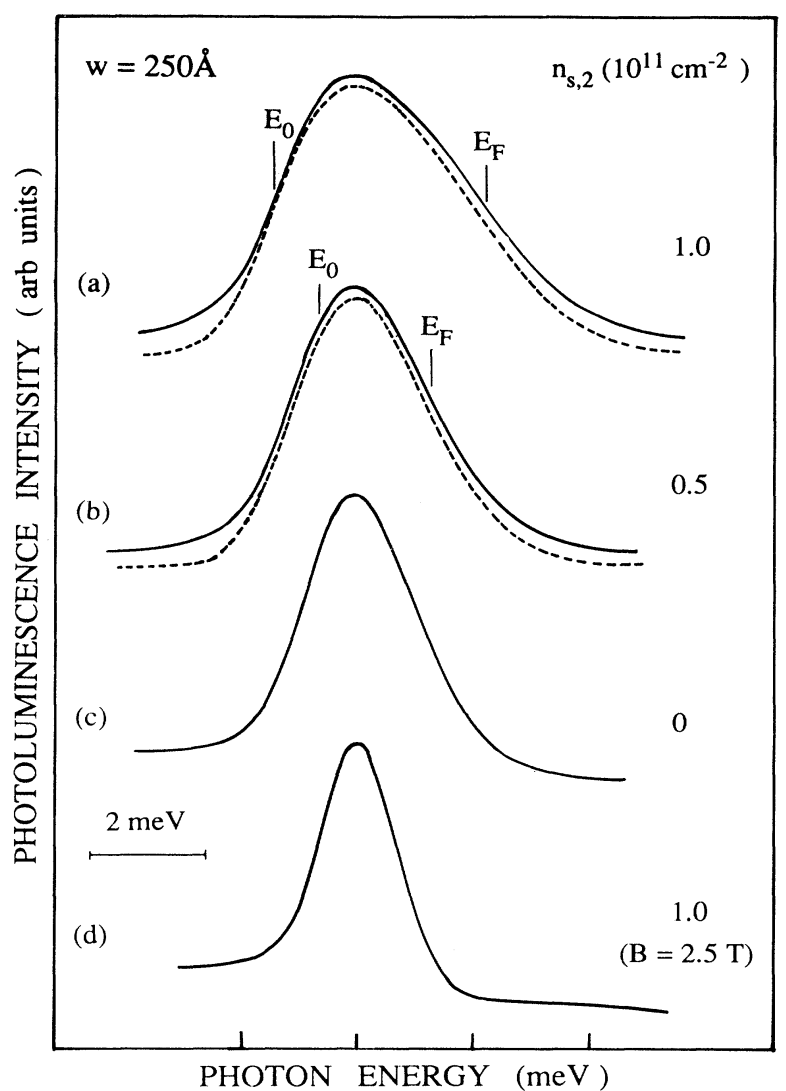

FIG. 5. Narrowing of the observed $E_{21}$ PL linewidth with decreasing $n_{s, 2}$ is shown (full line) for (a) the as-grown sample $A$ $\left(n_{s, 2} \simeq 1 \times 10^{11} \mathrm{~cm}^{-2}\right)$, and the gated sample at (b) $V_{b}=0 \mathrm{~V}$ $\left(n_{s, 2} \simeq 0.5 \times 10^{11} \mathrm{~cm}^{-2}\right)$, and (c) $V_{b}=0.6 \mathrm{~V}\left(\varepsilon_{F} \simeq E_{2}-E_{1}\right)$. The dashed lines in (a) and (b) show the line-shape simulations used to estimate $n_{s, 2}$ vs $V_{b}$. (d) The PL profile for the as-grown sample $A$ is shown at $B=2.5 \mathrm{~T}$, where only the lowest Landau level in $n=2$ is filled.

tive to $E_{11}$ at $T=4 \mathrm{~K}$. With increasing temperature the $E_{21}$ PL peak is seen to decrease significantly in amplitude relative to $E_{11}$ and broaden. In Fig. 7 the spectra obtained at similar temperatures are displayed for sample $C$ $\left(n_{s, 2}<0.3 \times 10^{11} \mathrm{~cm}^{-2}\right)$, in which temperature-dependent PLE studies identified the $E_{21}$ feature as being of atomic exciton nature. The peak height of $E_{21, X}$ in PL is only three times stronger than $E_{11}$ in this sample. As described previously the linewidth of the $n=2$ related transition $E_{21, X}(3.1 \mathrm{meV})$ is narrower than for sample $A(4.5$ meV) due to a much reduced carrier density in $n=2$. However, with increasing temperature the $E_{21, X}$ peak of sample $C$ also undergoes a similar reduction in amplitude, relative to $E_{11}$, and broadening.

The very similar behavior of the $n=2$ transitions in samples $A$ and $C$, despite the significant difference in $n=2$ population can be seen clearly in the quantitative plots of Figs. 8(a) and 8(b). The variation with temperature of the integrated PL intensity and PL peak ampli- 


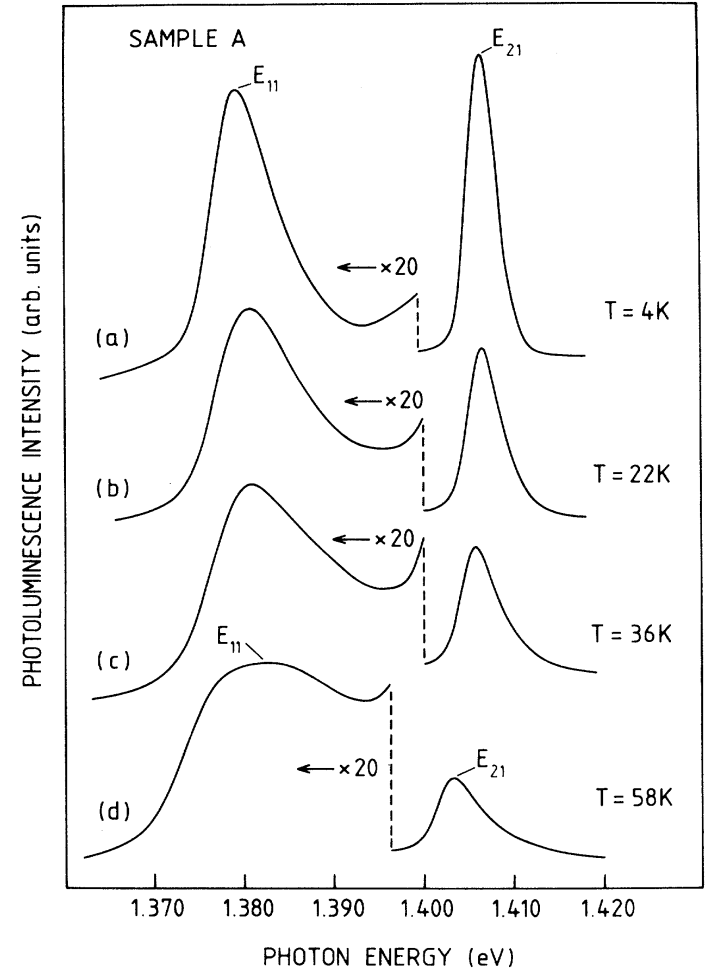

FIG. 6. PL spectra of sample $A$ showing the strong $E_{21}$ intensity, relative to $E_{11}(90 \%$ of the total intensity) at (a) $T=4$ $\mathrm{K}$. With increasing temperature, shown at (b) $T=22 \mathrm{~K}$, (c) $T=36 \mathrm{~K}$, and (d) $T=58 \mathrm{~K}$ the $E_{21}$ PL peak broadens and decreases in amplitude.

tude, respectively, of the $n=2$ related transitions (referred to generally as $E_{21}$ ) are plotted for samples $A$ (crosses), $B$ (circles), and $C$ (triangles). [Sample $B$ (PL) described in Sec. III) has a similar $n=2$ population to sample $C\left(n_{s, 2}<0.3 \times 10^{11} \mathrm{~cm}^{-2}\right)$. The $E_{21}$ PLE enhancement is of atomic exciton nature.] The intensity values of $E_{21}$ are normalized to the total integrated intensity of $E_{11}$ and $E_{21}$ at each temperature. The normalized values ratioed to the normalized values at $4 \mathrm{~K}$ are then plotted in Fig. 8(a). In a very similar way the $E_{21}$ peak amplitude normalized at each temperature to the $E_{21}$ integrated intensity, and then ratioed to the $4-\mathrm{K}$ values are plotted in Fig. 8(b).

For both types of sample ( $A: n_{s, 2} \simeq 1 \times 10^{11} \mathrm{~cm}^{-2}, B$, $C: n_{s, 2}<0.3 \times 10^{11} \mathrm{~cm}^{-2}$ ) the $E_{21}$ integrated PL intensity exhibits a rather weak decrease for $T \lesssim 20 \mathrm{~K}$. At $T \simeq 55$ $\mathrm{K}$ the $E_{21}$ intensity relative to $4 \mathrm{~K}$ has decreased to $\sim 0.80$ for sample $A$ and $\sim 0.68$ for sample $C$, shown in Fig. 8(a). By contrast the $E_{21}$ peak amplitudes [shown in Fig. 8(b)] decrease much more strongly for both types of samples, evident even at $T \simeq 20 \mathrm{~K}$. At $T \simeq 20 \mathrm{~K} E_{21}$ peak heights, relative to $4-\mathrm{K}$ values, of $\sim 0.65$ for both samples $A$ and $C$ are found; corresponding values of $\sim 0.45$ and $\sim 0.35$ near $55 \mathrm{~K}$ are found.

In order to understand these variations in $E_{21}$ intensity

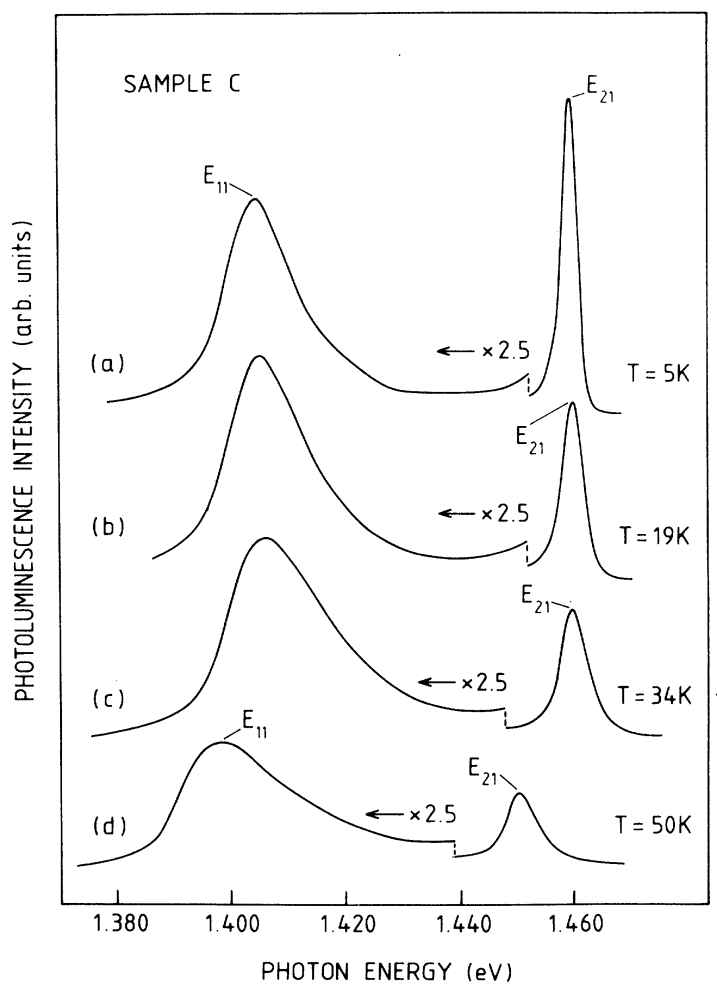

FIG. 7. PL spectra of sample $C$ where the $E_{21}$ PL is only $38 \%$ of the total intensity at (a) $T=5 \mathrm{~K}$. With increasing temperature, shown at (b) $T=19 \mathrm{~K}$, (c) $T=34 \mathrm{~K}$, and (d) $T=50 \mathrm{~K}$ the $E_{21}$ PL peak broadens and decreases in amplitude.

with temperature it must be understood that any changes in oscillator strength (or recombination rate) of $E_{21}$ as temperature is varied will only affect the $E_{21}$ intensity if there are competing recombination pathways of significant strength. The only important competing pathway of relevance is $E_{11} \mathrm{PL}$ recombination; nonradiative processes are eliminated by the normalization, described previously. In any case, the small changes in total PL intensity with temperature, even at $T=50 \mathrm{~K}$, for both types of sample, indicate that nonradiative recombination is not important. (At $T=35 \mathrm{~K}$ the total PL intensity relative to $4 \mathrm{~K}$ is 0.86 for sample $A$ and 0.95 for sample $C$.)

In sample $A$, with significant $n=2$ population $\left(n_{s, 2}=1 \times 10^{11} \mathrm{~cm}^{-2}\right)$, the $E_{21}$ PL intensity is dominant (90\% of the total PL intensity at $T=4 \mathrm{~K}$ ) principally due to the 10 times larger overlap of the $n=2$ electron wave function, relative to the $n=1$ electron wave function, with the first heavy-hole wave function. Therefore, since the main competing recombination channel $E_{11}$ (recombination rate $R_{1}$ ) is weak any decrease in $E_{21}$ recombination rate $R_{2}(T)$ leads to far weaker corresponding changes in $E_{21}$ relative integrated intensity $\left[I_{21, \text { rel }}=R_{2}(T) /\left(R_{2}(T)+R_{1}\right)\right] . E_{21}$ optical transitions at $T=4 \mathrm{~K}$ are known to be enhanced in oscillator strength by a factor of 2 , due to the FEES, from the PLE experiments of Sec. IV. In such a sample we now show that the 


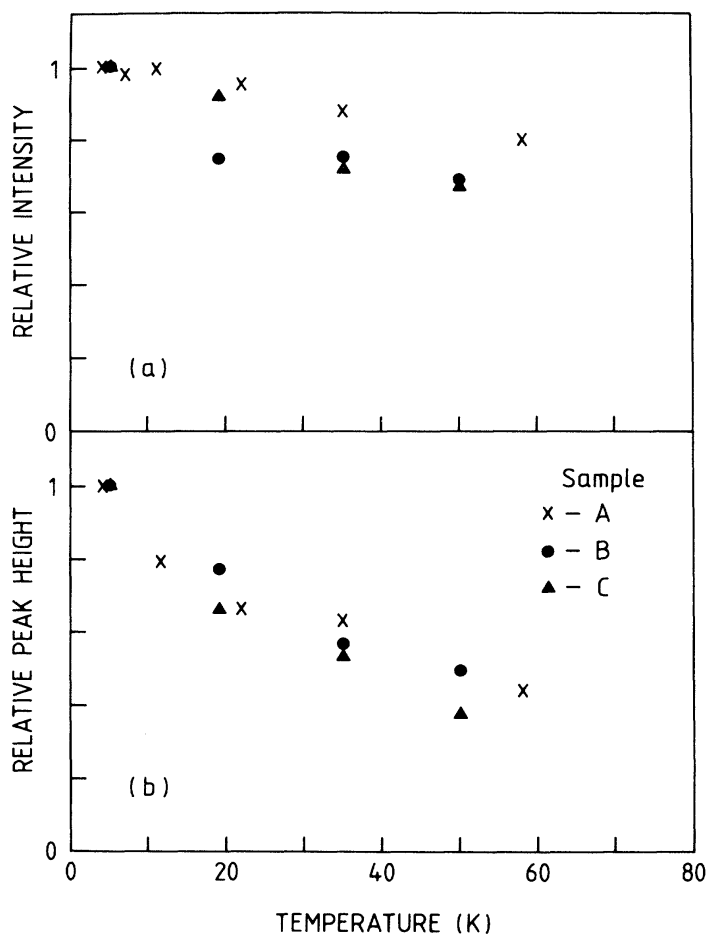

FIG. 8. The variations with temperature, relative to the $T=4 \mathrm{~K}$ values of (a) $E_{21}$ PL integrated intensity and (b) $E_{21}$ peak height for the two types of samples studied: sample $A$ (crosses) (FEES observed in PLE) and samples $B$ (circles) and $C$ (triangles) (no FEES observed in PLE). The intensity values of $E_{21}$ are normalized to the total integrated intensity of $E_{11}$ and $E_{21}$ at each temperature. The normalized values at $4 \mathrm{~K}$ are then plotted in (a). In Fig. (b) the $E_{21}$ peak amplitude is normalized at each temperature to the $E_{21}$ integrated intensity and then ratioed to the 4-K values. The temperature-dependent behavior is very similar for all samples.

decrease in intensity and peak height of $E_{21}$ with temperature can be understood without invoking the FEES mechanism.

The $E_{21}$ PL recombination involves band to band recombination between a Boltzmann thermal distribution of holes and a partially degenerate $n=2$ electron distribution. With increasing temperature the electron and hole population is spread out ${ }^{37}$ broadening the spectral range over which allowed $E_{21}$ band-to-band transitions occur. For any individual hole the probability of recombination with an electron is reduced, thus causing a weak reduction in the integrated intensity of the $E_{21}$ PL recombination, as observed [shown in Fig. 8(a) as crosses]. The spreading out of the electron and hole distribution also causes the corresponding decrease of the $E_{21}$ peak height with temperature. A line-shape calculation (similar to that described in Sec. IV) where the Fermi energy is adjusted to account for changes in thermal excitation of electrons into $n=2$, provides an estimate for the $E_{21}$ PL intensity and peak amplitude, relative to $T=4 \mathrm{~K}$. Values for the amplitude (normalized to the $T=4 \mathrm{~K} E_{21}$
PL intensity) of $0.75(T=20 \mathrm{~K})$ and $0.55(T=40 \mathrm{~K})$ are obtained from the line-shape simulation in reasonable agreement with the experimental value of 0.65 at $20 \mathrm{~K}$ and $\sim 0.60$ (interpolated) at $T=40 \mathrm{~K}$. The integrated PL intensity (normalized to the total PL intensity) is estimated on this model to decrease to $\sim 0.90$ at $T \sim 40$ $\mathrm{K},{ }^{38}$ in agreement with the experimental value of $\sim 0.90$, shown in Fig. 8(a).

In addition an extra factor of 2 reduction in oscillator strength of $E_{21}$ between 4 and $20 \mathrm{~K}$ is expected due to quenching of the FEES enhancement. ${ }^{10}$ However, since there are no strong competing pathways, only a small further reduction in relative intensity at $40 \mathrm{~K}$ (to 0.75 ) is expected. Therefore it is not possible by temperaturedependent PL measurements to distinguish clearly between FEES processes or band-to-band recombination.

For sample $C$ (where a FEES is not observed in PLE) the $E_{21}$ PL intensity is only $38 \%$ of the total PL intensity and $n_{s, 2}<0.3 \times 10^{11} \mathrm{~cm}^{-2}$. Since $n_{s, 2}$ is very low excitonic, rather than band to band, processes will be of greater importance for the $n=2$ recombination in these types of samples. (A similar discussion also applies to sample $B$ ). In this case the model we use for analyzing the temperature-dependent behavior of $E_{21}$ is based on that used by Feldmann et al. ${ }^{39}$ for $n=1$ excitons in undoped QW's. In undoped QW's the exciton recombination is restricted to a range of momentum states, $K_{\mathrm{ex}}$, about $K_{\text {ex }}=0$ over which the exciton oscillator strength is spread, corresponding to an exciton homogeneous spectral width of energy $\Delta(T) .{ }^{39,40}$ Elevated temperature causes an increase in $\Delta(T)$, where the overall magnitude of $\Delta(T)$ is determined by various coherence destroying processes, of which the temperature-dependent component is attributed to acoustic-phonon scattering. ${ }^{39,40}$ Such spreading contributes to a small increase in the (largely inhomogeneous) exciton linewidth and reduction in peak transition strength. Much more importantly, at higher temperatures $k T$ will be greater than $\Delta[\Delta(4 K) \lesssim 1$ $\mathrm{meV}$ typically]. Thus in (quasi) thermal equilibrium an increasing fraction, $1-r(T)$, of excitons occupy translational states $\left|K_{\mathrm{ex}}>0\right|$, which fall beyond the radiating momentum conserving range $\Delta(T)$. This redistribution results in reduced effective exciton oscillator strength with increasing temperature. ${ }^{39,40,41}$

Assuming that results valid for excitons in undoped QW's can be carried over to the present case, the fraction of excitons undergoing recombination, $r(T)$, is estimated to vary from $\sim 1$ to $\sim 0.35$ between $T \simeq 8$ and 40 K. Therefore, in the presence of competing $E_{11}$ band-toband recombination channels, a decreasing contribution from $E_{21, X}$ exciton recombination, can be expected with increasing temperature. Since the $E_{21}$ intensity is only a relatively small fraction of the total PL intensity (38\% in sample C) $E_{11}$ competing processes will contribute to the $E_{21}$ PL intensity to a much larger extent with increasing temperature than for sample $A\left(E_{21}\right.$ PL intensity $90 \%$ of total PL intensity). On the simplifying assumption that all $n=2$ recombination in sample $C$ is excitonic, then by $T \sim 40 \mathrm{~K}$ a reduction in $E_{21}$ intensity to $\sim 0.45$ of the 4 $\mathrm{K}$ value is expected, close to that observed [Fig. $8(\mathrm{~b})$ tri- 
angles]. In reality, at elevated temperatures band-toband processes are expected to assume increasing importance; ${ }^{37}$ thermal spreading of band-to-band transitions will then contribute additionally to the broadening and reduction in amplitude of the $E_{21} \mathrm{PL}$ peak, as for the case of sample $A$ above.

In summary the temperature dependence of PL from two types of samples has been presented here. One type (sample $A$ ) has strong $E_{21}$ PL involving a significant $n=2$ electron population $\left(n_{s, 2}=1 \times 10^{11} \mathrm{~cm}^{-2}\right)$ from which a FEES associated with $n=2$ is observed in PLE. The other type of sample ( $B$ and $C$ ) has $E_{F}$ close to $E_{2}$ $\left(n_{s, 2}<0.3 \times 10^{11} \mathrm{~cm}^{-2}\right)$ and in PLE an atomic exciton associated with $n=2$ is observed. Although the contribution of excitonic and band to band processes varies in the $E_{21}$ PL of the two types of samples, in contrast to the PLE results, the PL spectra converge to comparable form and intensity at higher temperatures $(T \gtrsim 40 \mathrm{~K})$.

Chen et al. ${ }^{21}$ have presented results for (AlGa)As(InGa)As-GaAs AMDQW's with 4-K spectra of similar appearance to samples $B$ and $C$, from which the broadening and decrease in amplitude of the $E_{21}$ PL peak with increasing $T$ was interpreted as evidence for a Fermi-energy edge singularity. However the results obtained on our samples show that a pronounced temperature dependence of $E_{21}$ transitions occurring close to $E_{F}$ in AMDQW's (where $E_{F} \simeq E_{2}-E_{1}$ ) can be interpreted in terms of changes in electron and exciton distributions alone. At elevated temperature, a broadened spectrum, generally characteristic of band to band recombination should result, as observed in samples $B$ and $C$ of the present experiment and Ref. 21. Therefore a pronounced temperature dependence of $E_{21}$ photoluminescence transitions does not provide conclusive evidence for a manybody enhancement of such transitions at low $T$ as suggested in Ref. 21. However if features due to both $E_{F}$ and $E_{21}$ are observed firm conclusions as to the manybody characteristics of the peak at $E_{F}$ can be reached. ${ }^{22,23}$

\section{CONCLUSION}

In conclusion, the many-body and atomic exciton features associated with transitions between $n=2$ electron and HH1 hole in AMDQW's have been studied. At zero magnetic field the temperature-dependent characteristics of the excitonic enhancement observed in the PLE of MDQW's with one or more occupied subbands have been shown to be controlled by the carrier density in the subband in which the enhancement occurs. For PLE transitions into the $n=2$ electron subband the strong broadening and weakening of the excitonic enhancement peak with temperature, the characteristic feature of the Fermi-energy edge singularity, is observed for $n=2$ carrier density above $\sim 0.4 \times 10^{11} \mathrm{~cm}^{-2}$.

It is only PLE (or absorption) measurements that can be used to provide clear optical evidence for the presence of a Fermi-energy edge singularity, since in this case true oscillator strengths are measured. By contrast in PL, competing pathways can have strong effects on the observed PL intensity. The pronounced reduction in $E_{21}$ PL peak height and increased broadening with temperature, can be accounted for in terms of thermal spreading of the electron or exciton population near the $n=2$ subband edge, and therefore does not provide unequivocal evidence for a many-body enhancement of the $E_{21}$ transition at low temperatures.

\section{ACKNOWLEDGMENTS}

Part of the experimental work was carried out at the Defense Research Agency (DRA), Malvern, U.K. (formerly RSRE). Two of us (T.A.F., P.E.S.) are grateful to N. A. McLean for expert technical assistance. We are grateful to K. J. Nash, J. M. Rorison, M. K. Saker, and D. M. Whittaker for informative discussions. One of us (P.E.S.) acknowledges financial support from the DRA and the Australian Research Council. Support from the Science and Engineering Research Council (UK) under Grant No. GR/HO8082 is also acknowledged gratefully.
${ }^{1}$ R. Dingle, H. I. Stormer, A. C. Gossard, and W. Wiegmann, Appl. Phys. Lett. 33, 665 (1978).

${ }^{2}$ A. Pinczuk, J. Shah, R. C. Miller, A. C. Gossard, and W. Wiegmann, Solid State Commun. 50, 735 (1984).

${ }^{3}$ C. Delalande, G. Bastard, J. Orgonasi, J. A. Brum, H. W. Liu, M. Voos, G. Weimann, and W. Schlapp, Phys. Rev. Lett. 59, 2690 (1987); H. Yoshimura, G. E. W. Bauer, and H. Sakaki, Phys. Rev. B 38, 10791 (1988).

${ }^{4}$ S. Schmitt-Rink, D. S. Chemla, and D. A. B. Miller, Adv. Phys. 38, 89 (1989).

${ }^{5}$ M. S. Skolnick, J. M. Rorison, K. J. Nash, D. J. Mowbray, P. R. Tapster, S. J. Bass, and A. D. Pitt, Phys. Rev. Lett. 58, 2130 (1987).

${ }^{6}$ G. Livescu, D. A. B. Miller, D. S. Chemla, M. Ramaswamy, T. Y. Chang, N. Sauer, A. C. Gossard, and J. H. English, IEEE J. Quantum Electron QE-24, 1677 (1988).

${ }^{7}$ M. K. Saker, M. S. Skolnick, P. A. Claxton, J. S. Roberts, and M. J. Kane, Semicond. Sci. Technol. 3, 691 (1988).

${ }^{8}$ J. S. Lee, N. Miura, and T. Ando, J. Phys. Soc. Jpn. 59, 2254
(1990).

${ }^{9}$ S. R. Andrews, A. S. Plaut, R. T. Harley, and T. M. Kerr, Phys. Rev. B 41, 5040 (1990).

${ }^{10}$ M. S. Skolnick, D. M. Whittaker, P. E. Simmonds, T. A. Fisher, M. K. Saker, J. M. Rorison, R. S. Smith, P. B. Kirby, and C. R. H. White, Phys. Rev. B 43, 7354 (1991).

${ }^{11}$ A. E. Ruckenstein, S. Schmitt-Rink, and R. C. Miller, Phys. Rev. Lett. 56, 504 (1986).

${ }^{12}$ S. Schmitt-Rink, C. Ell, and H. Haug, Phys. Rev. B 33, 1183 (1986).

${ }^{13}$ A. E. Ruckenstein and S. Schmitt-Rink, Phys. Rev. B 35, 7551 (1987).

${ }^{14}$ J. Gavoret, P. Nozières, B. Roulet, and M. Combescot, J. Phys. (Paris) 30, 987 (1989).

${ }^{15}$ R. Sooryakumur, D. S. Chemla, A. Pinczuk, A. C. Gossard, W. Wiegmann, and L. J. Sham, Solid State Commun. 54, 859 (1985); Y. C. Chang and G. D. Sanders, Phys. Rev. B 32, 5521 (1985).

${ }^{16}$ K. J. Nash, M. S. Skolnick, M. K. Saker, and S. J. Bass, Phys. 
Rev. Lett. 70, 31115 (1993).

${ }^{17}$ G. E. W. Bauer, Phys. Rev. Lett. 64, 60 (1990); H. Yoshimura and H. Sakaki, Surf. Sci. 229, 508 (1990).

${ }^{18}$ A. J. Tuberfield, S. R. Haynes, P. A. Wright, R. A. Ford, R. G. Clark, J. F. Ryan, J. J. Harris, and C. T. Foxon, Phys. Rev. Lett. 65, 637 (1990); A. J. Turberfield, R. A. Ford, I. N. Harris, J. F. Ryan, C. T. Foxon, and J. J. Harris, J. F. Ryan, C. T. Foxon, and J. J. Harris, Phys. Rev. B 47, 4794 (1993).

${ }^{19}$ D. Heiman, B. B. Goldberg, A. Pinczuk, C. W. Tu, A. C. Gossard, and J. H. English, Phys. Rev. Lett. 61, 605 (1988); B. B. Goldberg, D. Heiman, A. Pinczuk, L. Pfeiffer, and K. West, ibid. 65, 641 (1990); H. Buhmann, W. Joss, K. von Klitzing, I. V. Kukushkin, A. S. Plaut, G. Martinez, K. Ploog, and V. B. Timofeev, ibid. 66, 926 (1991).

${ }^{20}$ Y. Masumoto, M. Matsuura, S. Tarucha, and M. Okamoto, Phys. Rev. B 32, 4275 (1985); W. Masselink, P. J. Pearah, J. Klem, C. K. Peng, H. Morkoc, G. D. Sanders, and Y. C. Chang, Phys. Rev. B 32, 8027 (1985).

${ }^{21}$ W. Chen, M. Fritze, A. V. Nurmikko, D. Ackley, C. Colvard, and H. Lee, Phys. Rev. Lett. 64, 2434 (1990).

${ }^{22}$ W. Chen, H. Fritze, A. Nurmikko, M. Hong, and L. L. Chang, Phys. Rev. B 43, 14738 (1991).

${ }^{23}$ W. Chen, M. Fritze, W. Walecki, A. V. Nurmikko, D. Ackley, J. M. Hong, and L. L. Chang, Phys. Rev. B 45, 8464 (1992).

${ }^{24}$ M. S. Skolnick, P. E. Simmonds, and T. A. Fisher, Phys. Rev. Lett. 66, 963 (1991); W. Chen, M. Fritze, and A. V. Nurmikko, ibid. 66, 964 (1991).

25J. F. Mueller, Phys. Rev. B 42, 11189 (1990); J. F. Mueller, A. Ruckenstein, and S. Schmitt-Rink, Mod. Phys. Lett. 2, 135 (1991).

26P. Hawrylak, Phys. Rev. B 44, 3821 (1991); 44, 6262 (1991).

${ }^{27}$ P. Hawrylak, Phys. Rev. B 42, 8986 (1990).

${ }^{28}$ T. A. Fisher, P. E. Simmonds, M. S. Skolnick, D. M. Whittaker, R. S. Smith, and N. A. McLean, Surf. Sci. 267, 528 (1992).

${ }^{29}$ P. B. Kirby, J. A. Constable, and R. S. Smith, Phys. Rev. B
40, 3013 (1989).

${ }^{30}$ G. Ji, D. Huang, U. K. Reddy, T. S. Henderson, R. Houdre, and H. Morkoc, J. Appl. Phys. 62, 3366 (1987).

${ }^{31}$ C. Colvard, N. Nouri, H. Lee, and D. Ackley, Phys. Rev. B 39, 8033 (1989).

${ }^{32}$ S. K. Lyo and E. D. Jones, Phys. Rev. B 38, 4113 (1988).

${ }^{33}$ M. S. Skonick, K. J. Nash, M. K. Saker, S. J. Bass, P. A. Claxton, and J. S. Roberts, Appl. Phys. Lett. 50, 1885 (1987).

${ }^{34}$ M. S. Skolnick, D. G. Hayes, P. E. Simmonds, A. W. Higgs, G. W. Smith, H. J. Hutchinson, C. R. Whitehouse, L. Eaves, M. Henini, O. H. Hughes, M. L. Leadbeater, and D. P. Halliday, Phys. Rev. B 41, 10754 (1990).

${ }^{35}$ M. S. Skolnick, K. J. Nash, P. R. Tapster, D. J. Mowbray, S. J. Bass, and A. D. Pitt, Phys. Rev. B 35, 5925 (1987).

${ }^{36}$ J. Christen and D. Bimberg, Phys. Rev. B 42, 7213 (1990).

${ }^{37}$ M. Colloci, M. Guriola, A. Vinaltieri, J. Appl. Phys. 68, 2809 (1990).

${ }^{38} \mathrm{~A}$ reduction in $E_{21}$ oscillator strength of approximately a factor of 2 is calculated due to the spreading of the electron and hole occupancies from 4 to $40 \mathrm{~K}$. Since $I_{21, \text { rel }}=R_{2}(T) /\left[R_{2}(T)+R_{1}\right]$ and $R_{2}(4 \quad \mathrm{~K}) \simeq 9 R_{1}(4 \mathrm{~K}) \quad$ a reduction in $R_{2}$ by a factor of 2 from 4 to $40 \mathrm{~K}$ leads to $I_{21 \text {, rel }}$ $(40 \mathrm{~K})=0.91 I_{21, \text { rel }}(4 \mathrm{~K})$. As discussed in the text since $E_{21}$ recombination is the dominant recombination pathway a factor of 2 reduction in $E_{21}$ oscillator strength leads to only a small change in $E_{21}$ intensity.

${ }^{39}$ D. Feldmann, G. Peter, E. O. Gobel, P. Dawson, K. Moore, C. T. Foxon, and R. J. Elliott, Phys. Rev. Lett. 59, 2337 (1987).

${ }^{40}$ C. C. Phillips, R. Eccleston, and S. R. Andrews, Phys. Rev. B 40, 9760 (1989).

${ }^{41}$ In high-quality undoped QW's with high quantum efficiency and hence no competing recombination pathways, such changes lead chiefly to increased lifetimes, with little change in PL intensity Ref. 39. 